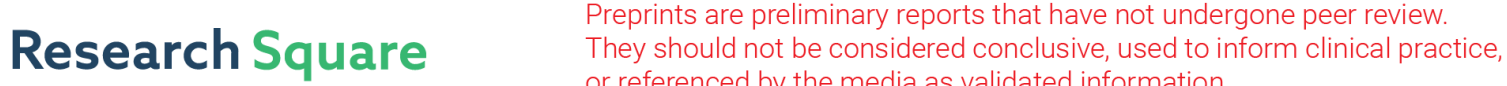 or referenced by the media as validated information. \\ Differentially Private Density Estimation with Skew- Normal Mixtures Model
}

Weisan Wu ( $\nabla$ wuws009@outlook.com )

Northeast Normal University

Research Article

Keywords: Density, DP-MSNM, Estimation

Posted Date: December 31st, 2020

DOI: https://doi.org/10.21203/rs.3.rs-134360/v1

License: (c) (i) This work is licensed under a Creative Commons Attribution 4.0 International License.

Read Full License 


\title{
Differentially Private Density Estimation with Skew-Normal Mixtures Model
}

\author{
Weisan $\mathrm{Wu}^{1,2, *}$
}

\author{
${ }^{1}$ Key Laboratory for Applied Statistics of MOE and School of Mathematics and Statistics,Northeast Normal \\ University, Changchun,130024, China \\ ${ }^{2}$ School of Mathematics and Statistics,Baicheng Normal University, Baicheng,137000, China \\ *wuws009@outlook.com
}

\begin{abstract}
The protection of private data is a hot research issue in the era of big data. Differential privacy is a strong privacy guarantees in data analysis. In this paper, we propose DP-MSNM, a parametric density estimation algorithm using Multivariate Skew-Normal Mixtures (MSNM) model to differential privacy. MSNM can solve the asymmetric problem of datasets, and it is could approximate any distribution through Expectation-Maximization (EM) algorithm. In this model,we add two extra steps on the estimated parameters in the M step of each iteration. The first step is adding calibrated noise to the estimated parameters based on Laplacian mechanism. The second step is post-processes those noisy parameters to ensure their intrinsic characteristics based on the theory of vector normalize and positive semi definition matrix. Extensive experiments using both real datasets evaluate the performance of DP-MSNM, and demonstrate that the proposed method outperforms DP-GMM.
\end{abstract}

\section{Introduction}

With the rapid developments of big data technology, data privacy protection has become a important issue. Differential privacy is an attack - resistant model with strict theoretical proof and good quantitative representation. Differential private density estimation has been done on Mixtures Model. The most of them are considered in the Gaussian Mixtures Model(GMM) ${ }^{12}$,but the data can present skewness or heavy tailed behavior. Nearly, the vulnerability of pre-trained models to the attack is not uniform when the training data itself is skewed, Trues (2019) shows that risk from some attacks is routinely increased when models use skewed training data ${ }^{3}$. This risk may be caused by the reduction of the accuracy of the model density estimation on assumed data normality.

To the best of our knowledge no existing work has concern in the Skew-Normal Mixtures model with differential privacy. In Skew-Normal mixtures model, all data are assumed to be drawn by randomly sampling from one of component of the Skew-Normal mixture model, and the learning task is to estimate the proportion parameters, location parameters, scale parameters and skewness parameters of each component distribution in model. In particular, we need to consider some problem of Skew-Normal mixtures model, where (i) G Skew-Normal distribution compose the Skew-Normal mixtures model, and(ii) we not clear the sample belongs to which component, and (iii) we just only have perturbed data,and (iv) we hope to get estimate values of all parameters.

Addressing on these problems, we propose DP-MSNM, which appends two extra steps (noise adding step and postprocessing step) to the general method of density estimation with MSNM in each iteration like. The noise adding step is adding classical Laplace noise to the original estimated parameters in each iteration to achieve differential privacy. The post-processing step will through eigen decomposition on scale parameter to prevent its not positive semi-definite and use normalize method to ensure sum the weight parameter as to 1 .

The remainder of the paper is organized as follows. Section 2 introduces preliminaries. Given DP-MSNM details in Section 3. Section 4 evaluates the experimental performance. Finally, Section 5 concludes the work and discusses future works.

\section{Preliminaries}

Let $D$ be a dataset that contains $n$ records $x_{1}, \cdots, x_{n}$ with $p$ attributes, and $x_{i}=\left(x_{i 1}, \cdots, x_{i p}\right)$. There $\|x\|_{p},\|A\|_{p}$ are denote the $L_{p}$ norms of vector $x$ and matrix $A$.

\section{Differential privacy}

The privacy definition is due to Dwork et al.(2006) ${ }^{4}$. The inform definition is given follows. 
Definition 1. (Differential Privacy) A randomized algorithm mechanism $\mathscr{M}$ provides $\varepsilon$-differential privacy, iffor all Output $O$ of $\mathscr{M}$ and for any two neighbor datasets $D$ and $D^{\prime}$, we have

$$
P(\mathscr{M}(D)=O) \leq e^{\varepsilon} P\left(\mathscr{M}\left(D^{\prime}\right)=O\right)
$$

We called $\varepsilon$ as privacy budget, the smaller $\varepsilon$ means the higher level of privacy provides. Where we adding calibrated random noise to the output to achieved privacy. We use Laplacian mechanism and $L_{1}$ sensitivity to output perturbation.

Definition 2. ( $L_{1}-$ sensitivity) The $L_{1}$ sensitivity of a query function $\mathscr{M}$ as the maximum change in the $L_{1}-$ norm of the query output on the neighbor datasets $D$ and $D^{\prime}$.That is ,

$$
S(\mathscr{M})=\max _{d\left(D, D^{\prime}\right)=1}\left\|\mathscr{M}(D)-\mathscr{M}\left(D^{\prime}\right)\right\|_{1}
$$

We denote the noisy vector or matrix are follows Laplace distribution $\operatorname{Lap}\left(0, \frac{S(\mathscr{M})}{\varepsilon}\right)$,

where $\frac{S(\mathscr{M})}{\varepsilon}$ is scale parameter. Given $L_{1}$ sensitivity $S(\mathscr{M})$ and the privacy budget $\varepsilon$, this mechanism can ensure differential privacy.

\section{Density Estimation with MSNM}

In the Multivariate Skew-Normal mixture models(MSNM), we assumed sample $x_{i}$ is i.i.d. with a density $f_{M S N}\left(x_{i}\right)$ that can be written as a linear composition of Skew-Normal distributions in the form

$$
\begin{aligned}
f_{M S N M}(x ; \Theta) & =\sum_{k=1}^{G} \pi_{k} f_{M S N}\left(x ; \theta_{k}\right) \\
& =\sum_{k=1}^{G} \pi_{k}\left\{2 \phi_{d}\left(x ; \xi_{k}, \Sigma_{k}, \alpha_{k}\right) \Phi\left(\alpha_{k}^{T} \sigma^{-1}\left(x-\xi_{k}\right)\right)\right\},
\end{aligned}
$$

where $x=\left(x_{1}, \cdots, x_{n}\right)$, and $x_{i}, i=1, \cdots, n$ is a $p$-dimensional vector. $\Theta=\left(\theta_{1}, \cdots, \theta_{G}\right), \theta_{k}=\left(\xi_{k}, \Sigma_{k}, \alpha_{k}\right), k=1, \cdots, G$. where $\pi_{k} \geq 0$ is mixture proportion and satisfied that $\sum_{k=1}^{G} \pi_{k}=1 . \xi_{k}, \Sigma_{k}, \alpha_{k}$ are parameter in the $k$-th component of the mixture models, and $\alpha_{k}$ is the $p$-dimensional skewness parameter, $\Sigma_{k}$ is a $p \times p$ positive semi-definite(PSD) correlation matrix, $\xi_{k}$ is the $p$-dimensional local parameter. $\phi_{d}(x ; \xi, \Sigma)$ is the density of a normal distribution $N(\xi, \Sigma), \Phi(\cdot)$ is the cumulate distribution function of standard normal. The function $f_{M S N}(x ; \xi, \Sigma, \alpha)$ can be present by

$$
f_{M S N}(x ; \xi, \Sigma, \alpha)=2 \phi_{d}(x ; \xi, \Sigma, \alpha) \Phi\left(\alpha^{T} \sigma^{-1}(x-\xi)\right)
$$

where $\sigma=\left(\Sigma \odot I_{p}\right)^{\frac{1}{2}}, \odot$ denote Hadamard product. If a random variable $X$ has density function like equation (2), we called the variable $\mathrm{X}$ is a Multivariate Skew-Normal distribution, and denote $X \sim S N_{p}(\xi, \Sigma, \alpha)$.

Recalling the variables $X_{0} \sim S N_{p}(0, \bar{\Sigma}, \alpha)$ and $X \sim S N_{p}(\xi, \Sigma, \alpha)$, we can obtain an additional representation for Multivariate Skew-Normal distribution from the results of Azzalini et al. ${ }^{5}$ as follows:

$$
X_{0}=D_{\delta} Z_{0}+\delta\left|Z_{1}\right|,
$$

where $\delta=\left(\delta_{1}, \delta_{2}, \cdots, \delta_{p}\right)^{T}$ is a vector with the elements in $(-1,1)$, and

$$
D_{\delta}=\left(I_{p}-\operatorname{diag}(\delta)^{2}\right)^{1 / 2},
$$

$U_{0}$ and $U_{1}$ are the normal variables of dimension $p$ and 1 , the joint distribution is

$$
\left(\begin{array}{c}
Z_{0} \\
Z_{1}
\end{array}\right) \sim N_{p+1}\left(0,\left(\begin{array}{cc}
\bar{\Omega} & 0 \\
0 & 1
\end{array}\right)\right)
$$

$\bar{\Psi}$ is a full-rank correlation matrix.

By the transformation $X=\xi+\sigma X_{0}$, we have

$$
Y=\xi+\sigma X_{0}=\xi+\sigma D_{\delta} Z_{0}+\sigma \delta\left|Z_{1}\right| .
$$

Let $\left|Z_{1}\right|=\tau$. Given $\tau$, we can obtain the condition representation, that is,

$$
X \mid \tau \sim N_{p}\left(\xi+\sigma \delta \tau, \sigma D_{\delta} \bar{\Omega} D_{\delta} \sigma\right) .
$$


For a $p$-dimensional SN distribution, the parameters have the following relationships by simple algebraic work $\left(\right.$ See $\left.^{5}\right)$ :

$$
\begin{aligned}
& \omega=D_{\delta}^{-1} \delta ; \\
& \bar{\Sigma}=D_{\delta}\left(\bar{\Omega}+\omega \omega^{T}\right) D_{\delta} ; \\
& \alpha=\left(1+\omega^{T} \bar{\Omega}^{-1} \omega\right)^{-1 / 2} D_{\delta}^{-1} \bar{\Omega}^{-1} \omega ; \\
& \delta=\left(1+\alpha^{T} \bar{\Sigma} \alpha\right)^{-1 / 2} \bar{\Sigma} \alpha .
\end{aligned}
$$

Furthermore, we have

$$
\bar{\Sigma}=D_{\delta} \bar{\Omega} D_{\delta}+D_{\delta} \omega \omega^{T} D_{\delta}=D_{\delta} \bar{\Omega} D_{\delta}+\delta \delta^{T} .
$$

Thus, let $\eta=\sigma \delta$, by the relation between $\Sigma$ and $\bar{\Sigma}$, we have

$$
\Sigma=\sigma \bar{\Sigma} \sigma=\sigma D_{\delta} \bar{\Omega} D_{\delta} \sigma+\sigma \delta \delta^{T} \sigma=\sigma D_{\delta} \bar{\Omega} D_{\delta} \sigma+\eta \eta^{T} .
$$

Therefore

$$
X \mid \tau \sim N_{p}\left(\xi+\sigma \delta \tau, \Sigma-\eta \eta^{T}\right) .
$$

Let $Z_{i k}$ be the membership indicator variable such that it equals 1 when $x_{i}$ is from the $k$-th component of the MSNM model, and equals 0 otherwise. Consider the complete data $(X, Z)=\left\{X_{i}, Z_{i}\right\}_{i=1}^{n}$, where the latent component-indicators vector $Z_{i}=\left(Z_{1 i}, \cdots, Z_{G i}\right)$ follows a multi-nomial distribution with the trial and cell probabilities of $\pi_{1}, \cdots, \pi_{G}$. Let us write it as $Z_{i} \sim M\left(1 ; \pi_{1}, \cdots, \pi_{G}\right)$. Based on the component indicators, for each $X_{i}, i=1, \cdots, n$, the hierarchical representation for MSNMs can be given by

$$
\begin{aligned}
& X_{i} \mid \tau_{i}, Z_{i k}=1 \sim N_{p}\left(\xi_{k}+\sigma_{k} \delta_{k} \tau_{i}, \Sigma_{k}-\eta_{k} \eta_{k}^{T}\right), \\
& \tau_{i} \mid Z_{i k}=1 \sim T N_{[0,+\infty)}(0,1), \\
& Z_{i} \sim \operatorname{Multi}\left(1 ; \pi_{1}, \cdots, \pi_{G}\right),
\end{aligned}
$$

where $T N_{[0,+\infty)}(0,1)$ denotes the half normal distribution. According to the hierarchical representation (6), ignoring the added constants and denoting

$$
\Lambda_{k}=\Sigma_{k}-\eta_{k} \eta_{k}^{T}
$$

the complete data log-likelihood function is

$$
\begin{aligned}
p \ell_{n}^{c}(\Theta)= & \sum_{i=1}^{n} \sum_{k=1}^{G} Z_{i j}\left\{\log \left(\pi_{k}\right)-\frac{1}{2} \log \left|\Lambda_{k}\right|-\frac{1}{2}\left(x_{i}-\xi_{k}\right)^{T} \Lambda_{k}^{-1}\left(x_{i}-\xi_{k}\right)\right. \\
& \left.+\left(x_{i}-\xi_{k}\right)^{T} \Lambda_{k}^{-1} \eta_{k} \tau_{i}-\frac{1}{2} \eta_{k}^{T} \Lambda_{k}^{-1} \eta_{k} \tau_{i}^{2}-\frac{1}{2} \tau_{i}^{2}\right\}
\end{aligned}
$$

According to the Bayesian theorem, we have

$$
\tau_{i} \mid\left(X_{i}=x_{i}, Z_{i k}=1\right) \sim T N_{[0,+\infty)}\left(\mu_{\tau_{i k}}, \sigma_{\tau_{i k}}^{2}\right),
$$

where

$$
\mu_{\tau_{i k}}=\frac{\left(x_{i}-\xi_{k}\right)^{T} \Lambda_{k}^{-1} \eta_{k}}{1+\eta_{k}^{T} \Lambda_{k}^{-1} \eta_{k}}, \quad \sigma_{\tau_{i k}}^{2}=\frac{1}{1+\eta_{k}^{T} \Lambda_{k}^{-1} \eta_{k}}
$$

Thus, for the current parameters $\Theta^{(t)}$, let

$$
\mu_{\tau_{i k}}^{(t)}=\frac{\left(x_{i}-\xi_{k}^{(t)}\right)^{T}\left(\Lambda_{k}^{(t)}\right)^{-1} \eta_{k}^{(t)}}{1+\eta_{k}^{T}\left(\Lambda_{k}^{(t)}\right)^{-1} \eta_{k}^{(t)}}, \quad \sigma_{\tau_{i k}}^{2(t)}=\frac{1}{1+\left(\eta_{k}^{(t)}\right)^{T}\left(\Lambda_{k}^{(t)}\right)^{-1} \eta_{k}^{(t)}} .
$$

The EM algorithm then proceeds as follows: 
E-step: Let us compute the conditional expectations as

$$
\begin{aligned}
r_{0 i k}^{(t)} & =\frac{\pi_{k}^{(t)} f_{M S N}\left(x_{i} ; \xi_{k}^{(t)}, \Sigma_{k}^{(t)}, \alpha_{k}^{(t)}\right)}{\sum_{j=1}^{G} \pi_{j}^{(t)} f_{M S N}\left(x_{i} ; \xi_{j}^{(t)}, \Sigma_{j}^{(t)}, \alpha_{j}^{(t)}\right)}, \\
r_{1 i k}^{(t)} & =E\left(\tau_{i} \mid X_{i}=x_{i}, Z_{i k}=1, \Theta^{(t)}\right)=\mu_{\tau_{i k}}^{(t)}+\sigma_{\tau_{i k}}^{(t)} \Delta_{i k}^{(t)}, \\
r_{2 i k}^{(t)} & =E\left(\tau_{i}^{2} \mid X_{i}=x_{i}, Z_{i k}=1, \Theta^{(t)}\right)=\mu_{\tau_{i k}}^{2(t)}+\sigma_{\tau_{i k}}^{(t)}+\mu_{\tau_{i k}}^{(t)} \sigma_{\tau_{i k}}^{(t)} \Delta_{i k}^{(t)},
\end{aligned}
$$

where $\Delta_{i k}^{(t)}=\phi\left(\frac{\mu_{\tau_{i k}}^{(t)}}{\sigma_{\tau_{i k}}^{(t)}}\right) / \Phi\left(\frac{\mu_{\tau_{i k}}^{(t)}}{\sigma_{\tau_{i k}}^{(t)}}\right)$. Thus, we get $E\left(Z_{i k} \tau_{i} \mid X_{i}=x_{i}, \Theta^{(t)}\right)=r_{0 i k}^{(t)} r_{1 i k}^{(t)}$, and $E\left(Z_{i k} \tau_{i}^{2} \mid X_{i}=x_{i}, \Theta^{(t)}\right)=r_{0 i k}^{(t)} r_{2 i k}^{(t)}$. Therefore, the $\mathrm{Q}$ function can be written as

$$
\begin{aligned}
Q\left(\Theta, \Theta^{(t)}\right)= & \sum_{i=1}^{n} \sum_{k=1}^{G} r_{0 i k}^{(t)}\left\{\log \left(\pi_{k}\right)-\frac{1}{2} \log \left|\Lambda_{k}\right|-\frac{1}{2}\left(x_{i}-\xi_{k}\right)^{T} \Lambda_{k}^{-1}\left(x_{i}-\xi_{k}\right)\right. \\
& \left.+\left(x_{i}-\xi_{k}\right)^{T} \Lambda_{k}^{-1} \eta_{k} r_{1 i k}^{(t)}-\frac{1}{2}\left(1+\eta_{k}^{T} \Lambda_{k}^{-1} \eta_{k}\right) r_{2 i k}^{(t)}\right\}
\end{aligned}
$$

M-Step: Let us maximize $Q\left(\Theta, \Theta^{(t)}\right)$ with respect to $\Theta$ under the restriction with $\sum_{k=1}^{G} \pi_{k}=1$.

1. Update $\pi_{k}^{(t)}$ by $\pi_{k}^{(t+1)}=n^{-1} \sum_{i=1}^{n} r_{0 i k}^{(t)}$.

2. Update $\xi_{k}^{(t)}$ by

$$
\xi_{k}^{(t+1)}=\frac{\sum_{i=1}^{n} r_{0 i k}^{(t)} x_{i}-\eta_{k}^{(t)} \sum_{i=1}^{n} r_{0 i k}^{(t)} r_{1 i k}^{(t)}}{\sum_{i=1}^{n} r_{0 i k}^{(t)}}
$$

3. Update $\eta_{k}^{(t)}$ by

$$
\eta_{k}^{(t+1)}=\frac{\sum_{i=1}^{n} r_{0 i k}^{(t)}\left(x_{i}-\xi_{k}^{(t+1)}\right) r_{1 i k}^{(t)}}{\sum_{i=1}^{n} r_{0 i k}^{(t)}}
$$

4. Update $\Lambda_{k}^{(t)}$ by

$$
\begin{aligned}
\Lambda_{k}^{(t+1)}= & \frac{1}{\sum_{i=1}^{n} r_{0 i k}^{(t)}}\left\{\sum_{i=1}^{n} r_{0 i k}^{(t)}\left(x_{i}-\xi_{k}^{(t+1)}\right)\left(x_{i}-\xi_{k}^{(t+1)}\right)^{T}\right. \\
& \left.-2 \eta_{k}^{(t+1)} \sum_{i=1}^{n} r_{0 i k}^{(t)} r_{1 i k}^{(t)}\left(x_{i}-\xi_{k}^{(t+1)}\right)+\eta_{k}^{(t+1)} \sum_{i=1}^{n} r_{0 i k}^{(t)} r_{2 i k}^{(t)}\left(\eta_{k}^{(t+1)}\right)^{T}\right\} .
\end{aligned}
$$

\section{Differentially private MSNM}

In this section, we present DP-MSNM method which is a differentially private density estimation algorithm with MSNM.

\section{Main idea}

Let $G$ denotes the component order of the MSNM. The main idea of DP-MSNM is add to two extra steps after getting the original estimated parameters in the M-step of each iteration. The original estimated parameters include mixture proportion $(\pi)$, local parameter vector $\left(\xi_{k}\right)$, scale parameter matrix $\left(\Sigma_{k}\right)$ and skewness parameter vector $\left(\alpha_{k}\right)$ of each Skew-Normal distribution, where $\pi=\left(\pi_{1}, \cdots, \pi_{G}\right)$ and $k \in\{1, \cdots, G\}$. First,we need to get noise of each parameter by $L_{1}$-sensitivity and allocated privacy budget, and add these noises to the original estimated parameters. We get noisy parameters $\bar{\pi}$ and $\bar{\xi}_{k}, \bar{\Lambda}_{k}, \bar{\eta}_{k}$. The second step is post-processes $\bar{\pi}$ and $\bar{\xi}_{k}, \bar{\Lambda}_{k}, \bar{\eta}_{k}$, since the noise added will break some intrinsic characteristics of weight parameter and component parameters,this step will output $\hat{\pi}$ and $\hat{\xi}_{k}, \hat{\Lambda}_{k}, \hat{\eta}_{k}$ 


\section{Noise adding step}

In this part, we will analyze the $L_{1}$ sensitivities of $\pi_{k}, \xi_{k}, \Lambda_{k}, \eta_{k}$, and we will add calibrated noise to the original estimated parameters. We suppose that $D=\left\{x_{1}, \cdots, x_{n}\right\}$ and $D^{\prime}=\left\{x_{1}, \cdots, x_{n-1}\right\}$ are two neighbor datasets, where $D^{\prime}$ has the same $n-1$ records as $D^{\prime}$, but not have the $n$-th record. Let $N_{k}=\sum_{i=1}^{n} r_{0 i k}$, we also assume the dataset is well-separated ${ }^{6}$ and $N_{k} \geq \frac{n}{2 G}, k=1, \cdots, G$. We use differentially private k-means algorithm ${ }^{7}$ to get the centers of clusters, and get $\eta_{k}$ form skew parameter $\alpha_{k}$ through Mardia measure method ${ }^{8}$. We denote $S(\pi), S(\xi), S(\Lambda), S(\eta)$ as the $L_{1}$ sensitivities of $\pi, \xi_{k}, \Lambda_{k}, \eta_{k}$ in each iteration. According to equation (8)(9)(10)(11), we give $S(\pi), S(\xi), S(\Lambda), S(\eta)$ in Lemma1,2,3,4.

Lemma 1. In each iteration, the $L_{1}$ sensitivity of $\pi$ is $S(\pi)=\frac{G}{n}$.

Lemma 2. In each iteration, if $\left\|x_{i}\right\|_{1} \leq R, N_{k} \geq \frac{n}{2 G}$ and $\left\|\eta_{k}\right\|_{1} \leq R_{3}$, the $L_{1}$ sensitivity of $\xi_{k}$ is $S(\xi)=\frac{4 G\left(R-R_{3}\right)}{n}$.

Proof: Since $\pi=\left(\pi_{1}, \cdots, \pi_{G}\right)$, we let $\xi(D)$ and $\xi\left(D^{\prime}\right)$ denote the local parameter of datasets $D$ and $D^{\prime}$. The k-th elements of $\xi(D)-\xi\left(D^{\prime}\right)$ is

$$
\begin{aligned}
& \frac{\sum_{i=1}^{n} r_{0 i k} x_{i}-\eta_{k} \sum_{i=1}^{n} r_{0 i k} r_{1 i k}}{\sum_{i=1}^{n} r_{0 i k}}-\frac{\sum_{i=1}^{n-1} r_{0 i k} x_{i}-\eta_{k} \sum_{i=1}^{n-1} r_{0 i k} r_{1 i k}}{\sum_{i=1}^{n-1} r_{0 i k}} \\
= & \frac{r_{0 n k} \sum_{i=1}^{n-1} r_{0 i k}\left(x_{n}-x_{i}\right)-\eta_{k} r_{0 n k} \sum_{i=1}^{n} r_{0 i k}\left(r_{1 n k}-r_{1 i k}\right)}{\sum_{i=1}^{n} r_{0 i k} \sum_{i=1}^{n-1} r_{0 i k}}
\end{aligned}
$$

Because of $\left\|x_{i}\right\|_{1} \leq R, N_{k} \geq \frac{n}{2 G},\left\|\eta_{k}\right\|_{1} \leq R_{3}$, we have

$$
\begin{aligned}
\left\|\xi(D)-\xi\left(D^{\prime}\right)\right\|_{1} & \leq \frac{2 G}{n} \frac{\left|R r_{0 n k} \sum_{i=1}^{n-1} r_{0 i k}-\eta_{k} r_{0 n k} \sum_{i=1}^{n-1} r_{0 i k}\right|}{\sum_{i=1}^{n-1} r_{0 i k}} \\
& \leq \frac{2 G}{n}\left(\left|R r_{0 n k}-\eta_{k} r_{0 n k}\right|\right) \\
& \leq \frac{4 G\left(R-R_{3}\right)}{n} .
\end{aligned}
$$

Lemma 3. In each iteration, if $\left\|x_{i}\right\|_{1} \leq R, N_{k} \geq \frac{n}{2 G}$ and $\left\|r_{1 i k}\right\|_{1} \leq R_{1},\left\|r_{2 i k}\right\|_{1} \leq R_{2}$, the $L_{1}$ sensitivity of $\eta_{k}$ is $S(\eta)=\frac{2 G R R_{1}}{n R_{2}}$.

Proof:let $\eta(D)$ and $\eta\left(D^{\prime}\right)$ denote the parameter in the equation (10) $D$ and $D^{\prime}$. The k-th elements of $\eta(D)-\eta\left(D^{\prime}\right)$ is

$$
\begin{aligned}
& \frac{\sum_{i=1}^{n} r_{0 i k}\left(x_{i}-\xi_{k}\right) r_{1 i k}}{\sum_{i=1}^{n} r_{0 i k} r_{2 i k}}-\frac{\sum_{i=1}^{n-1} r_{0 i k}\left(x_{i}-\xi_{k}\right) r_{1 i k}}{\sum_{i=1}^{n-1} r_{0 i k} r_{2 i k}} \\
= & \frac{r_{0 i k} \sum_{i=1}^{n-1} r_{0 i k}\left[r_{2 i k} r_{1 i k}\left(x_{n}-\xi_{k}\right)-r_{2 i k} r_{1 i k}\left(x_{i}-\xi_{k}\right)\right]}{\sum_{i=1}^{n} r_{0 i k} r_{2 i k} \sum_{i=1}^{n-1} r_{0 i k} r_{2 i k}}
\end{aligned}
$$

Then

$$
\left\|\eta(D)-\eta\left(D^{\prime}\right)\right\|_{1} \leq \frac{2 G R R_{1}}{n R_{2}} .
$$

Lemma 4. In each iteration, if $\left\|x_{i}\right\|_{1} \leq R, N_{k} \geq \frac{n}{2 G},\left\|r_{1 i k}\right\|_{1} \leq R_{1},\left\|r_{2 i k}\right\|_{1} \leq R_{2},\left\|\eta_{k}\right\|_{1} \leq R_{3}$, the $L_{1}$ sensitivity of $\Lambda_{k}$ is $S(\Lambda)=$ $\frac{2 G\left(R^{2}+4 R R_{1} R_{3}+4 R_{2} R_{3}^{2}\right)}{n}$.

Proof:let $\Lambda(D)$ and $\Lambda\left(D^{\prime}\right)$ denote the parameter in the equation (11) $D$ and $D^{\prime}$. The k-th element of vector $\Lambda(D)-\Lambda\left(D^{\prime}\right)$ is

$$
\begin{aligned}
& \frac{\sum_{i=1}^{n} r_{0 i k}\left(x_{i}-\xi_{k}\right)\left(x_{i}-\xi_{k}\right)^{T}-2 \eta_{k} \sum_{i=1}^{n} r_{0 i k} r_{1 i k}\left(x_{i}-\xi_{k}\right)^{T}+\eta_{k} \sum_{i=1}^{n} r_{0 i k} r_{2 i k} \eta_{k}^{T}}{\sum_{i=1}^{n} r_{0 i k}} \\
& -\frac{\sum_{i=1}^{n-1} r_{0 i k}\left(x_{i}-\xi_{k}\right)\left(x_{i}-\xi_{k}\right)^{T}-2 \eta_{k} \sum_{i=1}^{n-1} r_{0 i k} r_{1 i k}\left(x_{i}-\xi_{k}\right)^{T}+\eta_{k} \sum_{i=1}^{n-1} r_{0 i k} r_{2 i k} \eta_{k}^{T}}{\sum_{i=1}^{n-1} r_{0 i k}} \\
= & \frac{1}{\sum_{i=1}^{n} r_{0 i k} \sum_{i=1}^{n-1} r_{0 i k}}\left\{r_{0 n k} \sum_{i=1}^{n-1}\left[\left(x_{n}-\xi_{k}\right)\left(x_{n}-\xi_{k}\right)^{T}-\left(x_{i}-\xi_{k}\right)\left(x_{i}-\xi_{k}\right)^{T}\right]-\right. \\
& \left.2 \eta_{k} r_{0 n k} \sum_{i=1}^{n-1} r_{0 i k}\left[r_{1 n k}\left(x_{n}-\xi_{k}\right)^{T}-r_{1 i k}\left(x_{i}-\xi_{k}\right)^{T}\right]+\eta_{k} \sum_{i=1}^{n-1} r_{0 i k}\left[r_{0 n k} r_{2 n k} \eta_{k}^{T}-r_{0 n k} r_{2 i k} \eta_{k}^{T}\right]\right\}
\end{aligned}
$$


Then

$$
\left\|\Lambda(D)-\Lambda\left(D^{\prime}\right)\right\|_{1} \leq \frac{2 G\left(R^{2}+4 R R_{1} R_{3}+4 R_{2} R_{3}^{2}\right)}{n} .
$$

In each iteration, let $\varepsilon_{\pi}, \varepsilon_{\xi}, \varepsilon_{\Lambda}, \varepsilon_{\alpha}$ present the privacy budget allocated to $\pi, \xi_{k}, \Lambda_{k}, \alpha_{k}$ respectively, where $k=1, \cdots, G$. So, we know the privacy budget in each iteration is $\varepsilon_{\pi}+G\left(\varepsilon_{\xi}+\varepsilon_{\Lambda}+\varepsilon_{\alpha}\right)$ from composite theorem. Next we can get the noisy parameters $\bar{\pi}, \bar{\xi}_{k}, \bar{\Lambda}_{k}, \bar{\alpha}_{k}$ through Lemma 1,2,3,4 and the Laplace mechanism, it was given in follows:

$$
\begin{aligned}
\bar{\pi}_{k} & =\pi_{k}+e_{\pi_{k}}^{1 \times 1}, \\
\bar{\xi}_{k} & =\xi_{k}+e_{\xi_{k}}^{p \times 1}, \\
\bar{\Lambda}_{k} & =\Lambda_{k}+e_{\Lambda_{k}}^{p \times p}, \\
\bar{\eta}_{k} & =\eta_{k}+e_{\eta_{k}}^{p \times 1} .
\end{aligned}
$$

where $e_{\pi_{k}} \sim \operatorname{Lap}\left(0, \frac{S(\pi)}{\varepsilon_{\pi}}\right), e_{\xi_{k}} \sim \operatorname{Lap}\left(0, \frac{S(\xi)}{\varepsilon_{\xi}}\right), e_{\Lambda_{k}} \sim \operatorname{Lap}\left(0, \frac{S(\Lambda)}{\varepsilon_{\Lambda}}\right), e_{\eta_{k}} \sim \operatorname{Lap}\left(0, \frac{S(\eta)}{\varepsilon_{\eta}}\right)$ denote noise respect to $\pi_{k}, \xi_{k}, \Lambda_{k}, \eta_{k}$.

\section{Post-processing Step}

Because of $\bar{\pi}, \bar{\xi}_{k}, \bar{\Lambda}_{k}, \bar{\eta}_{k}$ would break some intrinsic characteristics in original parameter $\pi, \xi_{k}, \Sigma_{k}, \alpha_{k}$. Firstly, $\bar{\pi}$ not satisfied $\pi_{k} \geq 0$ and $\sum_{k=1}^{n}=1$ generally. Secondly, $\bar{\Lambda}_{k}$ would not be a positive semi-definite matrix after adding noisy. If the noisy covariance matrix $\bar{\Lambda}_{k}$ is not PSD matrix, the other parameters, for instance $\bar{\xi}_{k}, \bar{\alpha}_{k}$ can not be calculated exactly. We need to solve these problems as follow.

First, we use the method of normalized to the $\bar{\pi}$. We let $\bar{\pi}_{k}=\frac{\bar{\pi}_{k}-\min \{\bar{\pi}\}}{\max \{\bar{\pi}\}-\min \{\bar{\pi}\}}$, and make them sum to one by $\hat{\pi}_{k}=\frac{\bar{\pi}_{k}+\zeta}{\sum_{k=1}^{G} \bar{\pi}_{k}+G \zeta}$. There $\zeta$ is a small number, it can be smooth $\bar{\pi}$

Secondly, we can use theory of Higham $1988^{9}$ to ensure the covariance matrix $\bar{\Sigma}_{k}$ to be PSD through post-processing $\bar{\Lambda}_{k}$. Like Wu $2016^{1}$ we use the follow equation

$$
\hat{\Lambda}_{k}=\bar{\Lambda}_{k}+\rho\left(\bar{\Lambda}_{k}\right) I,
$$

where $\rho\left(\bar{\Lambda}_{k}\right)=\min \left\{r \geq 0: \bar{\Lambda}_{k}+r I \succeq 0\right\}, \succeq 0$ means that all the eigenvalues are not less than $0, I$ is the identity matrix. We use $\hat{\Lambda}_{k}$ to get $\hat{\xi}_{k}$, and next we will get $\hat{\eta}_{k}, \hat{\Sigma}_{k}$ and $\hat{\alpha}_{k}$ by equation (5)and (7).The parameters $\hat{\xi}_{k}, \hat{\Sigma}_{k}, \hat{\alpha}_{k}$ will be join into the next iteration calculation.

Although adding two extra steps on original parameters, we can know the parameters $\hat{\pi}, \hat{\xi}, \hat{\alpha}, \hat{\Sigma}$ also satisfied $\varepsilon$-differential privacy. ${ }^{10}$

\section{DP-MSNM}

From what discussed above, we give the DP-MSNM algorithm in Algorithm 2, where lines 1-2 allocate the privacy budget and initialize the parameters, lines 3 calculates the $L_{1}$-sensitivities of all parameters, lines 5-6 execute the normal E-step and M-step, lines 7-12 execute the noise adding step, lines 13-20 execute the post-processing step. Given total privacy budget $\varepsilon$, Wu $2016^{1}$ shows that under the maximum iteration $T$, Algorithm 2 satisfies $\varepsilon$-differential privacy. for $k=1, \cdots, G$, we allocate the privacy budget $\varepsilon_{\Lambda}=\frac{0.6}{T * G} \varepsilon$ to $\Lambda_{k}, \varepsilon_{\xi}=\frac{0.13}{T * G} \varepsilon$ and $\varepsilon_{\eta}=\frac{0.13}{T * G} \varepsilon$ to $\xi_{k}$ and $\eta_{k}$ respectively, because of the higher $\varepsilon_{\Lambda}$ can reduce the amount of noise added to the $\Lambda_{k}$.

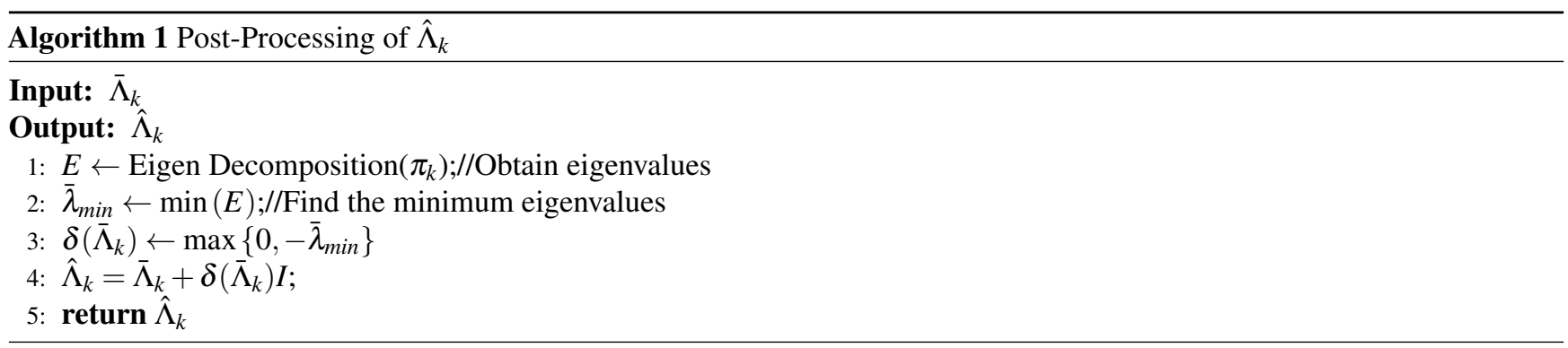

\section{Experimentral Evaluation}

Our experimental implemented in R based on mixsmsn package ${ }^{11}$ to get DP-MSNM results, and all experiments are performed on a laptop with Intel Core i7-9750H and $2.60 \mathrm{GHz}$ CPU and 6.00 RAM. 


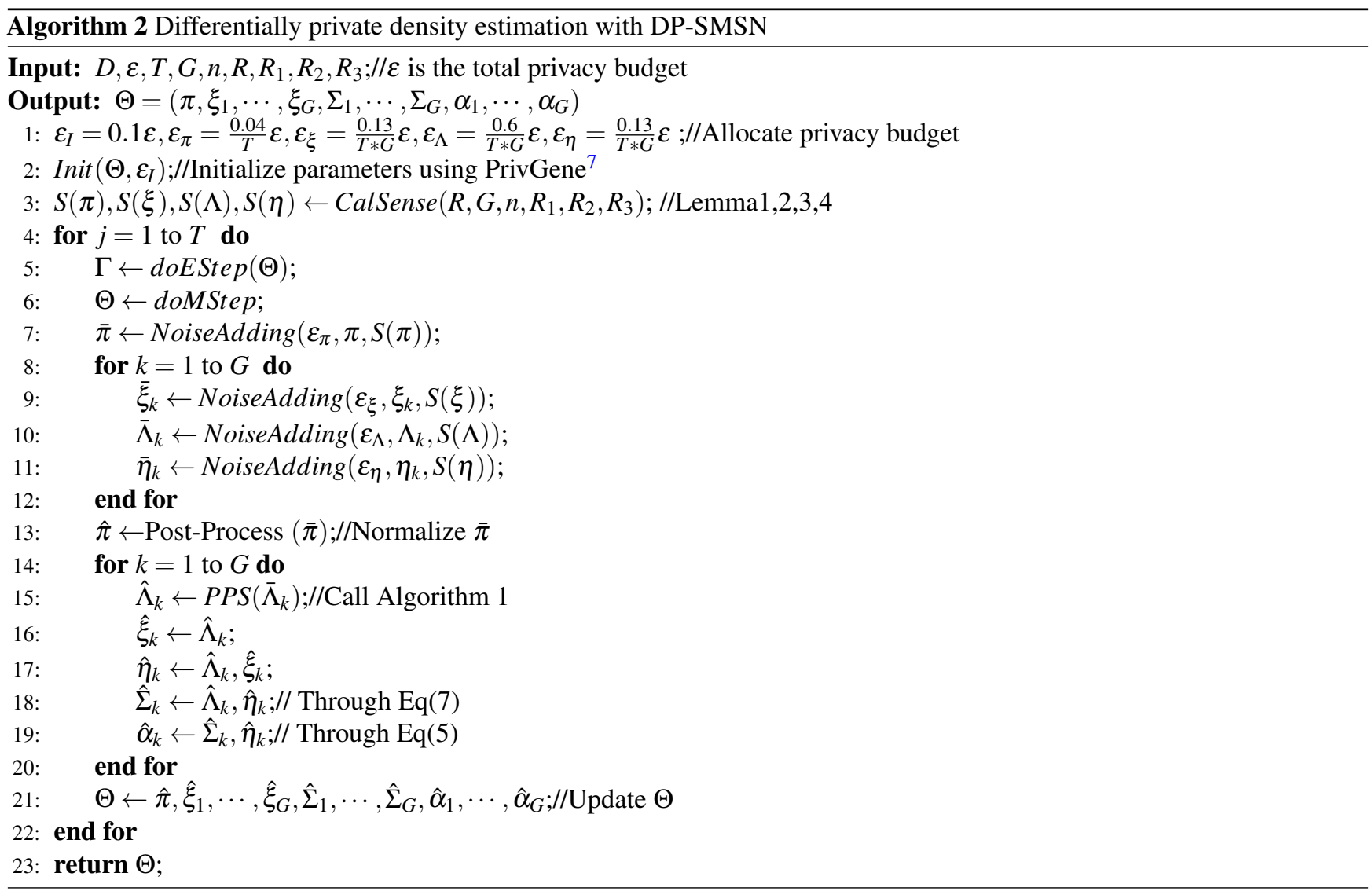

\section{Experimental setup}

We evaluate DP-MSNM on two real datasets, the Australian Institute of Sport dataset and the 3DRoad Network dataset, which from UCI Machine Learning Repository. The number of records in AIS dataset is 202 and the number of records in 3DRoad dataset is 434874. We choose the columns 2-3 in AIS dataset and the columns 2-4 in 3DRoad dataset.

We use $\log$-likelihood mean $\frac{1}{n} \log \left\{f_{M S N M}(x ; \pi, \xi, \Sigma, \alpha)\right\}$ which is the average log-likelihood of the whole dataset to evaluate the performance of DP-MSNM. The larger log-likelihood mean means that the better parameter estimation.

\section{Results}

\section{Compare with DP-GMM}

We implement DP-GMM by software R with the same environment as the DP-MSNM. From the Fig.1 and Fig.2 we find the DP-MSNM outperforms DP-GMM. Evaluation of DP-MSNM

Effect of Number of Skew-Normal Distribution(G)

From Fig.1, we can observe that in 3DRoad dataset, the log-likelihood mean has well effect when $k=6$. From Fig.2, we can see that in AIS dataset, the log-likelihood mean increases at first and starts decreasing when $k=7$. So, we can conclude that the order of the component has great effect to our results.

\section{Conclusions}

In this paper, we proposed DP-MSNM, which is a effective and accurate privacy-preserving mechanism to estimate density. We added noise to original parameters and through post-processing step to ensure weight and covariance parameter have it's intrinsic characteristics. In the future, we will extend the DP-MSNM model to high-dimensional data. We also plan to construct other Skew-family distributions to differential privacy.

\section{References}

1. Wu, Y., Wu, Y. \& Peng, H. Differentially private density estimation via gaussian mixtures model. IEEE/ACM 24th Int. Symp. 1-6 (2016). 


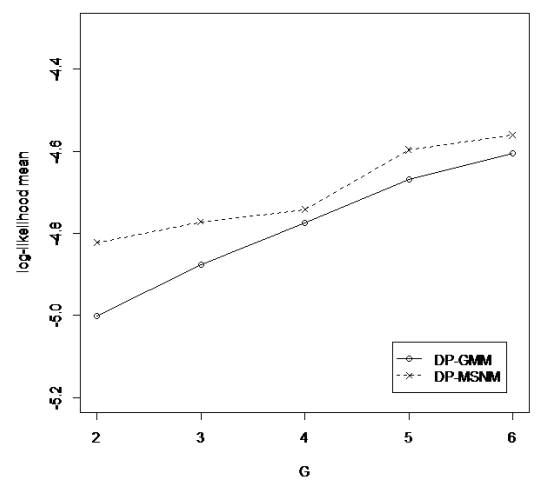

Figure 1. Effect of $\mathrm{G}$ in 3DRoad dataset

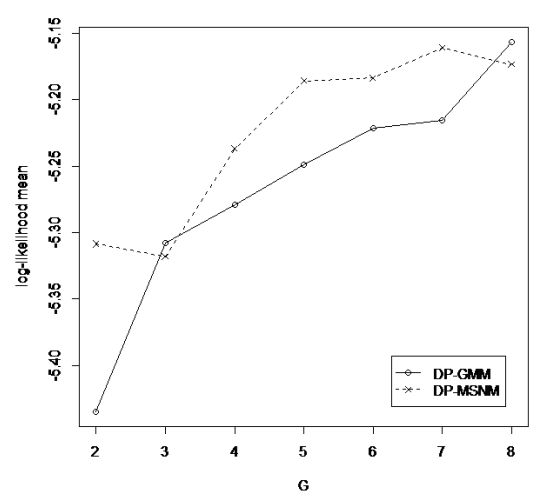

Figure 2. Effect of G in AIS dataset

2. Kamath, G., Sheffet, O. \& Singhal, V. Differentially private algorithms for learning mixtures of separated gaussians. NeurIPS 1-62 (2019).

3. Truex, S., Liu, L. \& Gursoy, M. Effects of differential privacy and data skewness on membership inference vulnerability. arXiv.org 1-16 (2019).

4. Dwork, C. \& Aaron, R. The algorithmic foundations of differential privacy. Foundations Trends Theor. Comput. Sci.9, 211-407 (2014).

5. A.Azzalini \& A.DallaValle. The multivariate skew-normal distribution. Biometrika 83, 715-726 (1996).

6. Nissim, K., Raskhodnikova, S. \& Smith, A. Smooth sensitivity and sampling in private data analysis. STOC 75-84 (2007).

7. Zhang, J., Xiao, X. \& Yang, Y. Privgene: Differentially private model fitting using genetic algorithms. SIGMOD 665-676 (2013).

8. V., M. K. Measures of multivariate skewness and kurtosis with applications. Biometrika 57, 519-530 (1970).

9. Higham, N. Computing a nearest symmetric positive semidefinite matrix. Linear Algebr. its Appl. 139, 103-118 (1988).

10. Hay, M., Rastogi, V. \& Miklau, G. Boosting the accuracy of differential private histograms through consistency. PVLDB 3, 1021-1032 (2010).

11. Cabral, C., Lachos, V. \& Prates, M. Multivariate mixture modeling using skew-normal independent distributions. Comput. Stat. Data Analysis 56, 126-142 (2012).

\section{Acknowledgements}

This work was supported by the National Natural Science Foundation of China (Grant No. 11701071).

\section{Author contributions statement}

Weisan $\mathrm{Wu}$ is the only author and wrote the manuscript text.

\section{Competing interests}

The author declares no competing interests.

\section{Additional information}

Correspondence and requests for materials should be addressed to W.W. 
Figures

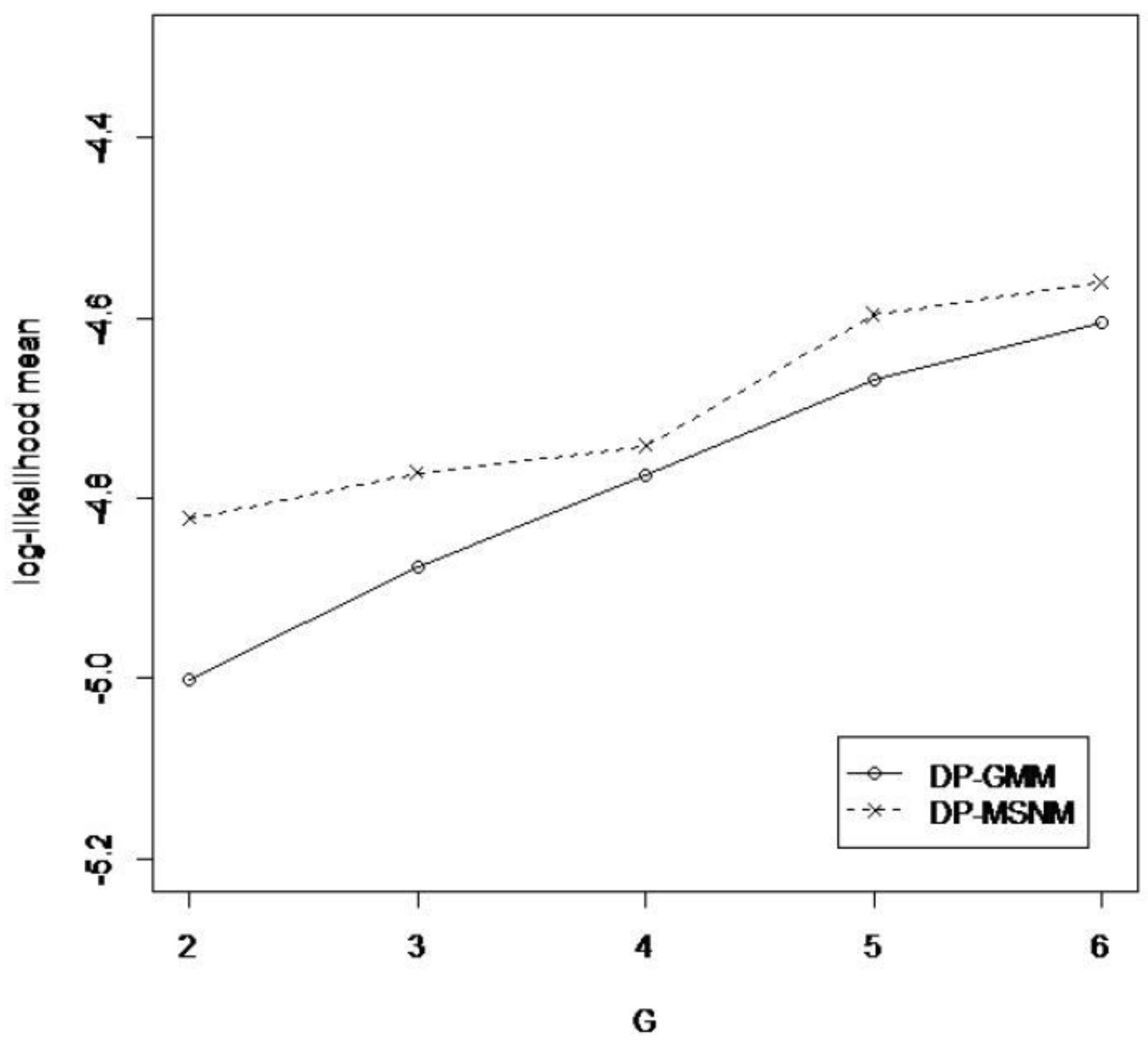

Figure 1

Effect of $G$ in 3DRoad dataset 


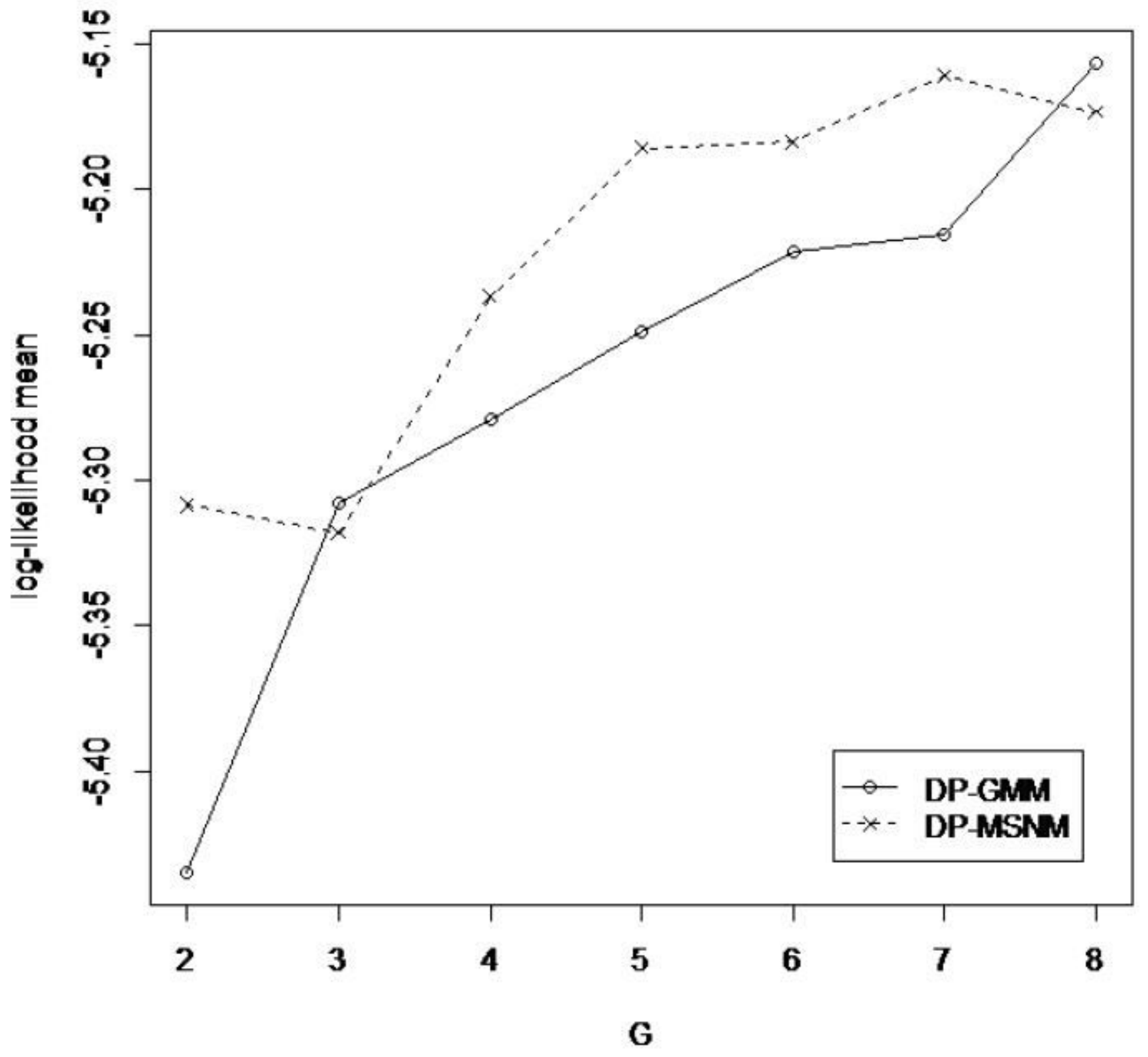

Figure 2

Effect of G in AIS dataset 